\title{
Activity-based therapies for repair of the corticospinal system injured during development
}

\section{Kathleen M. Friel ${ }^{1,2}{ }^{*}$, Preston T. J. A. Williams ${ }^{3}$, Najet Serradj $^{3}$, Samit Chakrabarty and John H. Martin ${ }^{3,5 *}$}

1 Department of Neurology, Brain and Mind Research Institute, Weill Cornell Medical College, New York, NY, USA

2 Burke Medical Research Institute, White Plains, NY, USA

${ }^{3}$ Department of Physiology, Pharmacology and Neuroscience, City College of the City University of New York, New York, NY, USA

${ }^{4}$ School of Biomedical Sciences, Faculty of Biology, University of Leeds, Leeds, UK

${ }^{5}$ The Graduate Center of the City University of New York, New York, NY, USA

\section{Edited by:}

Gavin John Clowry, Newcastle

University, UK

\section{Reviewed by:}

Claire Gibson, University of Leicester. UK

Ann-Christin Eliasson, Karolinska Institutet, Sweden

*Correspondence:

Kathleen M. Friel, Burke Medical Research Institution, White Plains, NY 10605, USA

e-mail: kaf3001@med.cornell.edu; John H. Martin, Department of Physiology, Pharmacology and Neuroscience, City College of the City University of New York, 160 Convent Avenue, New York, NY 10031, USA e-mail:jmartin@ccny.cuny.edu
This review presents the mechanistic underpinnings of corticospinal tract (CST) development, derived from animal models, and applies what has been learned to inform neural activity-based strategies for CST repair. We first discuss that, in normal development, early bilateral CST projections are later refined into a dense crossed CST projection, with maintenance of sparse ipsilateral projections. Using a novel mouse genetic model, we show that promoting the ipsilateral CST projection produces mirror movements, common in hemiplegic cerebral palsy (CP), suggesting that ipsilateral CST projections become maladaptive when they become abnormally dense and strong. We next discuss how animal studies support a developmental "competition rule" whereby more active/used connections are more competitive and overtake less active/used connections. Based on this rule, after unilateral injury the damaged CST is less able to compete for spinal synaptic connections than the uninjured CST. This can lead to a progressive loss of the injured hemisphere's contralateral projection and a reactive gain of the undamaged hemisphere's ipsilateral CST. Knowledge of the pathophysiology of the developing CST after injury informs interventional strategies. In an animal model of hemiplegic CP, promoting injured system activity or decreasing the uninjured system's activity immediately after the period of a developmental injury both increase the synaptic competitiveness of the damaged system, contributing to significant CST repair and motor recovery. However, delayed intervention, despite significant CST repair, fails to restore skilled movements, stressing the need to consider repair strategies for other neural systems, including the rubrospinal and spinal interneuronal systems. Our interventional approaches harness neural activity-dependent processes and are highly effective in restoring function. These approaches are minimally invasive and are poised for translation to the human.

Keywords: corticospinal tract, activity-dependent development, motor cortex, motor cortex stimulation, cerebral palsy, mirror movements

\section{INTRODUCTION}

The corticospinal (CS) system is the principal motor system in humans and many mammals for skilled movements. Damage to this system results in significant motor impairments. In maturity, loss of function after CS system injury, such as weakness and paresis, predominates. Essential coordination necessary for even the simplest of skilled movements, like reaching, grasping, and feeding, is also typically lost. Loss of motor skill and coordination are thought to be due primarily to the loss of the direct projections of the corticospinal tract (CST) from motor cortex to spinal cord motor circuits. CS system damage during development also leads to the gain of aberrant and debilitating functions that are key motor impairments in cerebral palsy (CP) (1). These include hyperreflexia and spasticity as well as aberrant limb and postural coordination. Mirror movements are also common in CP, particularly hemiplegic CP (2). Gain of aberrant functions in CP likely reflects several inter-related processes, including a loss of flexible and individuated muscle control replaced by relatively fixed motor synergies, and hyperreflexia and spasticity (3-6). Our research has identified another important factor contributing to impaired control - development of misprojections between spared cortical motor pathways and spinal and brain stem motor centers. This is maladaptive developmental miswiring of CS motor circuits, both of the injured system and the system that is spared. A critical question that we will focus on in this review is why the loss of CS connections due to perinatal brain trauma leads to maladaptive development of the surviving connections. Whereas it has been well-established that the brain is extraordinarily plastic early in development, beyond that there is a paucity of mechanisms to inform why miswiring occurs and how best to intervene. Our work in animals provides an understanding of the mechanisms underlying miswiring and a strategy to repair abnormal CS connectivity. 
In this review, we will first examine normal CS system development, including new findings on genetic developmental cues governing the laterality (i.e., contralateral or bilateral) of CST projections and motor function. Knowledge of the basic underlying genetic mechanisms of CST development can be leveraged to help inform why brain injury in $\mathrm{CP}$ can have a profound effect on wiring of the CST and the gain of aberrant functions. Next, we consider the role of neural activity-dependent processes in refinement of the CST from a bilateral to predominantly contralateral motor pathway. In the context of normal development, we briefly consider co-development of the CST and the rubrospinal system, the other major system for limb movement control. Limb movement normally reflects the dual actions of the cortical and rubrospinal systems $(7,8)$. Although we do not as yet know the specific reaction of the rubrospinal system to damage of the CS system, during the period when CS system damage leads to CP is when the rubrospinal system seems to play a unique role in limb control. Then, we will show how knowledge of normal development informs the question of why particular CST misprojections occur after early brain injury. Finally, we discuss how harnessing activity-dependent synaptic competition, which is key to normal development, leads to restoration of CST connections and recovery of motor skills in an animal model of hemiplegic CP. The aim of this review is to present the mechanistic underpinnings of motor system development derived from animal models, not to summarize therapeutic options for patients with CP. Nonetheless, toward the end of this review, we discuss strategies for neural repair based on our animal model that can be readily implemented in humans because they can be non-invasive or minimally invasive.

\section{GENETIC FACTORS HELP ESTABLISH THE LATERALITY OF CST SPINAL PROJECTIONS DURING DEVELOPMENT}

To understand why the CST develops misprojections in CP and how this relates to the motor impairments, we must examine first the mechanisms of normal development. As for other neural systems, the CST depends on the interplay between genetics, neural activity, and experience to achieve appropriate circuit formation and performance. Genetic mechanisms specify which cortical neurons develop to become CST neurons, and others develop to become interneurons and other projection neurons (9-11). Genetics also plays a key role in guiding CST axons to their targets in the spinal cord. Here, we focus on axon guidance because this is both important for normal wiring of connections and sets the stage for understanding why CST projections go awry after perinatal injury.

Diverse guidance molecules shepherd growing CST axons to their brain stem and spinal targets, including decussation of most CST axons from the medullary pyramid to the contralateral spinal cord white matter (12). CST neurons have complex sets of receptors that enable the guidance molecules present throughout the developing central nervous system to act as ligands to affect steering of the growth cone to ensure that CST axons reach their intended targets (13). The majority of CST axons reach the contralateral spinal segments after decussating in the pyramid and then project extensively into the gray matter on the same side as the descending spinal axons. This is the predominantly contralateral projection, characteristic of normal CST development.
In the spinal cord, the receptor tyrosine kinase EphA4, along with its ligand EphrinB3, restricts CST outgrowth from the contralateral to the ipsilateral spinal gray matter and thereby helps ensure a predominantly contralateral termination pattern (1417). EphA4 receptors on the CST growth cone bind to EphrinB3 on midline glial cells, which leads to axonal retraction. Evidence suggests that the overall level of EphA4 is used during development to regulate the amount of recrossing of the CST in the spinal cord and, in turn, the extent to which the developing CST establishes bilateral spinal terminations (17). In genetically normal animals - and likely in humans, as well - this mechanism results in a significant percentage of CST axons that project into the gray matter and recross within the spinal cord early in development. In the cat, which has been studied extensively, early-developing ipsilateral CST projections, which may be as much as $50 \%$ of the contralateral projection (18), are subsequently refined to a smaller number that is maintained into maturity (Figure 1). This refinement (discussed further below) establishes the predominantly contralateral CST projection pattern. Similarly, in normal human development, single-pulse transcranial magnetic stimulation (TMS) of motor cortex in neonates evokes bilateral responses, suggestive of strongly bilateral projections, whereas by 6 months of age, only contralateral responses are evoked $(19,20)$. The amount of recrossing also seems to vary across different animal species, with the monkey showing more than the cat or rodent $(21,22)$; although this has not been studied systematically.

Mouse genetics can be leveraged to study the question of development and maintenance of CST laterality, and importantly, inform the functional significance of bilateral CST projections for movement control. When the gene for EphA4 is eliminated selectively in the forebrain of mice, but not in the brain stem and spinal cord, the CST develops a strongly bilateral projection to the spinal cord (Figure 2A). These bilateral CST axons terminate on normally organized spinal circuits within the spinal cord $(16,17)$. An aberrant bilateral CST projection in mature mutant mice underlies robust bilateral CS motor circuit changes and bilateral voluntary behaviors. With bilateral CSTs, electrical stimulation of the motor cortex evokes bilateral limb muscle responses $(16,17)$, and a bilateral motor representation in motor cortex (17). In the knockout mouse, we characterized the motor map in terms of the number of sites where stimulation at threshold evoked mirror movements (identical movements of each forelimb). The wild type mice have no sites with this property, while approximately $80 \%$ of sites in all knockout mice evoked mirror movements (Figure 2B) (17). This shows that the aberrant bilateral CST projections in maturity are effective in activating spinal motor circuits bilaterally.

Does development of a bilateral CST and a mirror movement representation in motor cortex produce clear and consistent bilateral voluntary motor responses? This question was addressed by examining two motor behaviors that WT mice produce using unilateral limb movement: obstructed locomotion, where the animal steps over obstacles, and exploratory reaching (Figure 3). Both behaviors are voluntary. In obstructive locomotion, the animal uses obstacle sensory information (e.g., obstacle height and distance) to modify the gait pattern to clear the obstacle (23). And in exploratory reaching, the animal stands on its hind legs and reaches to explore the walls of the enclosure within which it is located (17). 


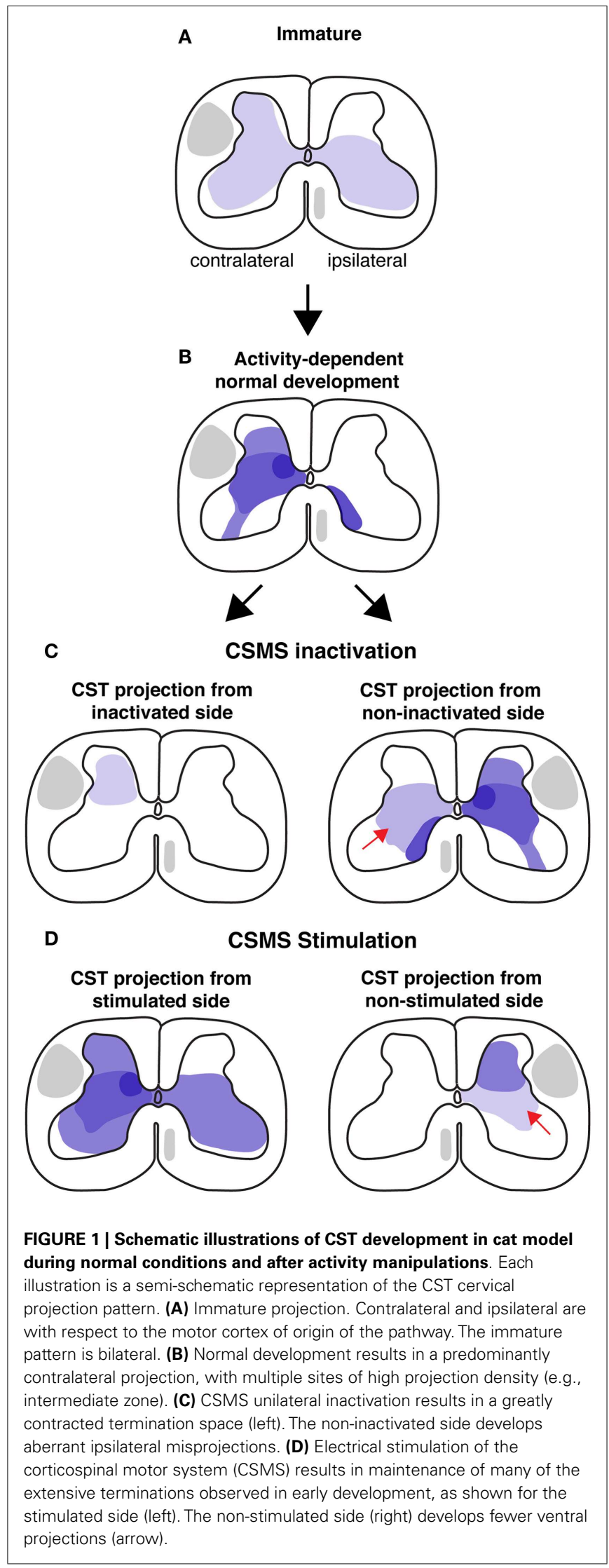

WT animals use alternate stepping over the obstacle and reach with one or the other forelimb (Figure 3). By contrast, EphA4 knockout mice rarely use alternate stepping to clear the obstacle, but instead use bilateral synchronous forelimb and hind leg movements, resembling hopping. During exploratory reaching, EphA4 knockout most commonly use both arms simultaneously, resembling mirror movements in people with CP (2). To summarize, by manipulating EphA4 signaling genetically we show the behavioral significance of ipsilateral CST misprojections (17). As we discuss below, ipsilateral CST projections become highly reactive after unilateral brain injury in CP. Similar to the EphA4 knockout mice, with a maladaptive expansion of the ipsilateral CST, many people with $\mathrm{CP}$ express mirror movements. Our findings using a genetic model further show that bilateral CST projections, not aberrant bilateral spinal circuits, can explain the presence of mirror movements.

\section{THE ACTIVITY OF THE MOTOR SYSTEMS AND LIMB USE REFINE CST PROJECTION PATTERNS}

Guidance molecules help to establish a coarse early spinal termination pattern of the CST. This pattern is subsequently refined later in development into the mature pattern (Figure 1). What is the mechanism by which the early coarse pattern of connections is refined? Since refinement occurs postnatally as the animal begins to express skilled motor behavior, we focused on the role of activity-dependent processes in establishment of the proper patterns of CST connections with spinal circuits. Our studies in the cat demonstrate an important role for the constitutive level of activity of the CS system in each hemisphere in establishment of spinal connections and a role for limb use, which likely reflects activity patterns. When activity in one motor cortex is blocked pharmacologically by infusing the $\mathrm{GABA}_{\mathrm{A}}$ agonist Muscimol during an early sensitive period, CST axons withdraw their projections [Figure 1C; left spinal cord; (24)]. Preventing use of one limb during a similar period has a similar effect on development of contralateral CST projections (25). By contrast, when electrically stimulated, CST axons extend more projections (Figure 1D; left spinal cord)(26). This shows the importance of activity-dependent factors in shaping the pattern of CST spinal projections and further suggests interactions - possibly competitive (see below) - between the CS systems from each hemisphere.

The unilateral activity interventions have major bilateral effects. The non-inactivated side (Figure 1C, right spinal cord) develops a normal contralateral projection but, additionally, an aberrant ipsilateral projection (Figure 1C, right, red arrow). Importantly, a bilateral CST from the less-affected side in often considered pathognomonic for hemiplegic CP (discussed below). Similarly, the non-stimulated side develops a diminished projection, with fewer intermediate and ventral projections (Figure 1D; bottom row, red arrow). These findings, together with more limited results from bilateral treatment [activity blockade; (27)], indicates the importance of the relative amount of activity in the developing CS system. We will see below that this is a key finding for understanding the pathophysiological mechanisms underlying $\mathrm{CP}$.

Thus, guidance mechanisms driven by genetic regulation of EphA4 initially help establish the density of ipsilateral CST axons. 


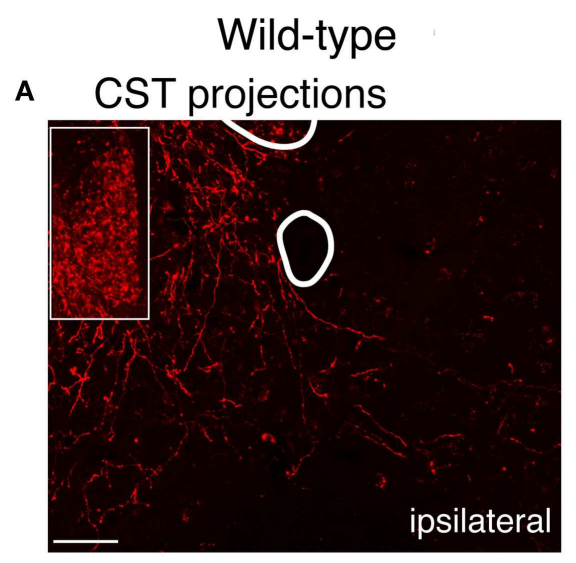

Emx1;EphA4tm2kidr

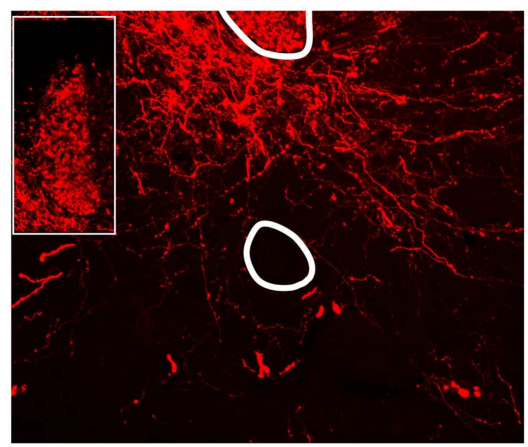

\section{B Motor cortex mirror movement sites}
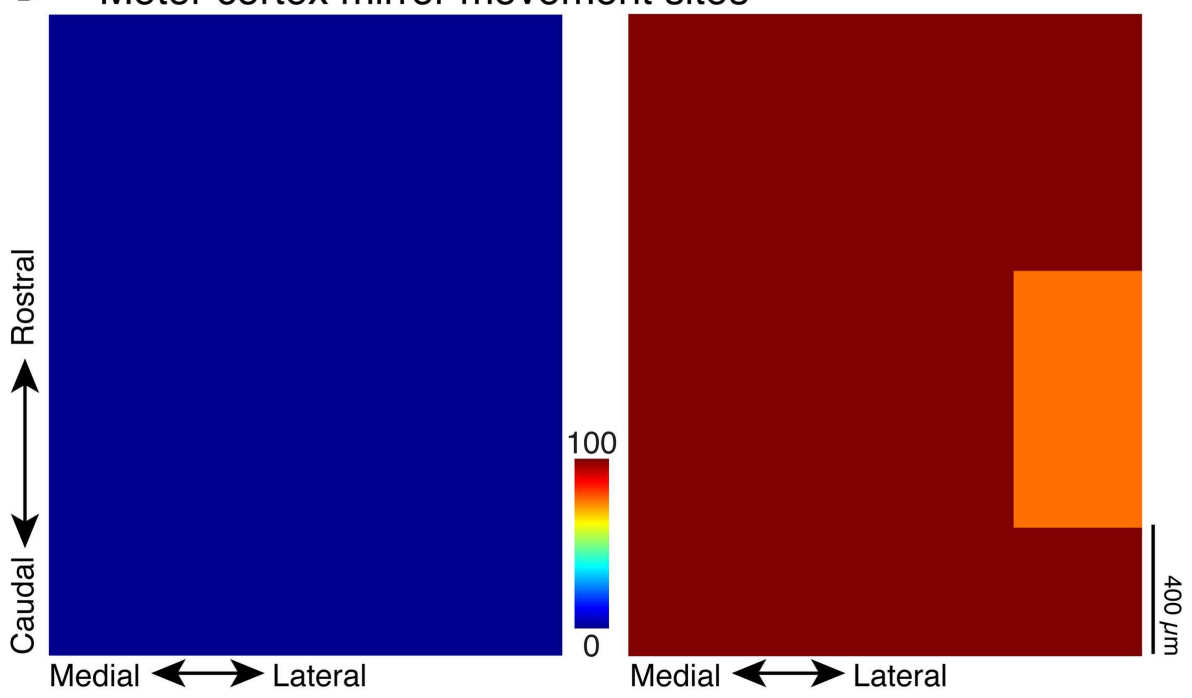

FIGURE 2 | CST in wild type and EphA4 conditional knockout mouse Modified from Serradj et al. (17). (A) CST terminations within the intermediate zone flanking the midline. Wild type mice (left) have a predominantly contralateral projection, whereas the mouse with conditional elimination of the EphA4 gene in the forebrain (right) has a strongly bilateral projection. The insets show labeling in the dorsal

Activity- and use-dependent processes subsequently shape the pattern initially established by genetic mechanisms. A plausible developmental strategy is that the more liberal the recrossing of CST axons, the greater opportunity for bilateral connections to be supported by early-developing bimanual limb movements. For example, if the developing child does not engage in extensive bimanual control, then more ipsilateral CST projections are eliminated compared with a child that uses more bimanual control. Further, the animal data provide support for a developmental rule whereby the more active (and more extensively used) connections of the developing CS system overtake less active/used connections. This helps explain the normal development of a predominantly crossed CST and the elimination of the early ipsilateral projections. As we discuss below, this activity-based rule also helps explain misprojections in $\mathrm{CP}$. column, which is the location of the CST in the mouse; labeling is comparable in the two mice. (B) Color-coded representations of sites in motor cortex where electrical stimulation evoked bilateral movements (e.g., mirror movements; average of five mice in each category). Whereas no "mirror sites" existed in the wild type mice (left), they were ubiquitous in the mutant mice (right).

\section{MOTOR REPRESENTATION DEVELOPMENT IN MOTOR CORTEX AND THE ROLE OF THE RED NUCLEUS IN EARLY MOVEMENT CONTROL}

The CST is one of many motor systems of the brain and spinal cord (28). In maturity, the CST functions together with the red nucleus (RN), which gives rise to the other major descending pathway for limb control, the rubrospinal tract $(7,8)$. The CS system is thought to play a greater role in more flexible and adaptive movements, and the rubrospinal system, in more automatic limb movements. Whereas the rubrospinal system has been speculated to play a role in recovery after CST damage in maturity $(29,30)$, its role in motor development in health and disease is not yet known (4). Interestingly, the rubrospinal system may have even more anatomical and functional prominence early in human development than it does later in life (31). We have 


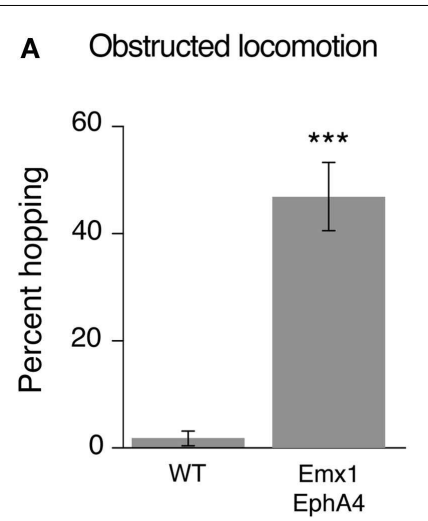

B Exploratory reaching

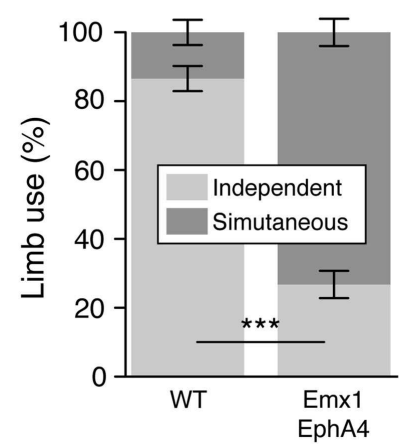

FIGURE 3 |The conditional EphA4 knockout mouse displays bilateral voluntary fore limb movements. Modified from Serradj et al. (17). (A) Obstructed locomotion. Wild type mice show alternate stepping whereas the EphA4 conditional mutant mice show synchronous fore limb movements (hopping) during obstructed locomotion. (B) Exploratory reaching. Wild type mice show independent limb use whereas the EphA4 conditional mutant mice show synchronous limb, or mirror movements, during reaching.

investigated development of the rubrospinal system in relation to CST development.

When the developing CS and rubrospinal systems are directly compared in an animal model, it has been shown that the rubrospinal system matures earlier than the CST (32). Early behavioral contributions of the CS and rubrospinal system can be evaluated by comparing development of their motor representations. The motor cortex motor map is a good indicator of CS system function (33). In the cat, for example, the motor cortex map comes "online" at about postnatal week 7 (Figure 4, dotted regression line) $(34,35)$ and this is when several measures of voluntary movement control - including object manipulation, and social/play interactions - develop $(36,37)$. Map development plateaus at about postnatal week 12, and this is also when expression of voluntary control stabilizes. Surprisingly, development of the red nucleus motor map begins earlier than the motor cortex motor map (Figure 4). In motor cortex, proximal limb joints are represented at younger ages then distal joints, also paralleling late development of distal skills (35). By contrast, in the red nucleus distal as well as proximal forelimb muscles are represented at the outset. Taken together, these findings suggest that the rubrospinal tract is important for establishing the rudiments of motor skills before the CST has come online.

An important question, yet to be resolved, is the extent to which these two motor systems interact during development. Emerging evidence from our laboratory suggests that there is an activitydependent interaction between the developing corticorubral and rubrospinal projections $(38,39)$. When motor cortex activity is blocked during early development, the red nucleus on the side of the inactivation may have an enhanced development, while development of the red nucleus motor map on the opposite side is remarkably impaired. This finding hints at a competition between the developing rubrospinal and CS systems. Red nucleus compensation on the injured side could be part of the biological

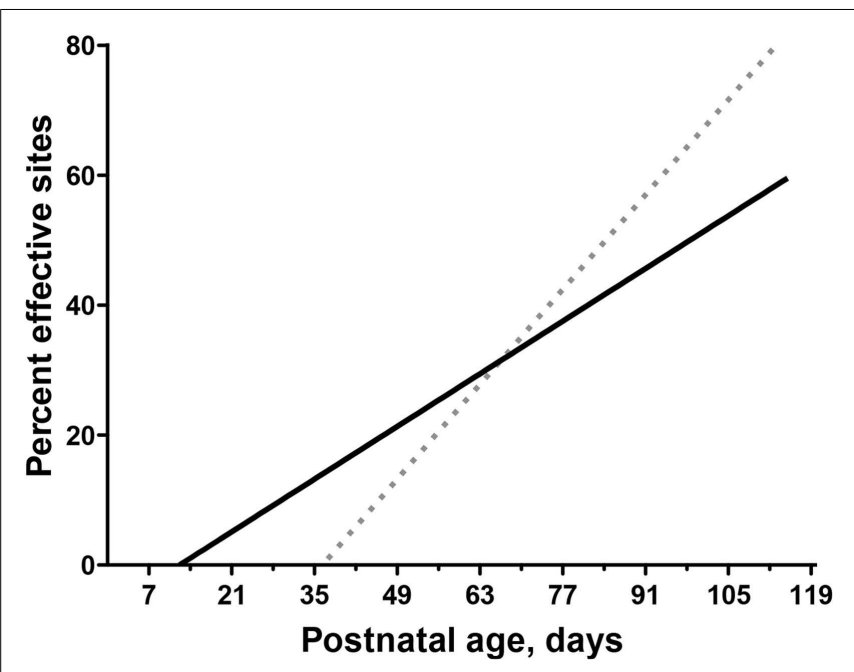

FIGURE 4 | Differential motor map development in motor cortex and red nucleus. Modified from Williams et al. (32) and Chakrabarty and Martin (35). Comparison of age-dependent increases in the percentage of sites in motor cortex (dotted line) and RN (solid line) where stimulation produced a contralateral motor response (termed effective sites). The linear fit is shown for both motor cortex $(Y=1.54 x-68.62)$ and $\mathrm{RN}(Y=0.66 x-10.03)$.

basis of partial recovery after developmental cortical injury. That the rubrospinal system precedes CS system development suggests that it may not be as prone to the same miswiring as the CST following early activity manipulations because its sensitive period is earlier.

\section{MECHANISMS OF MALADAPTIVE DEVELOPMENT OF THE CORTICOSPINAL SYSTEM AFTER UNILATERAL INJURY}

As expected, cortical and white matter injury destroys the cells of origin and connections of the CS system. In doing so, injury will not only disrupt CST function but system-wide development as well. With a predominantly unilateral injury, what is not well understood is why damage on one side leads to development of misprojections of the spared CST from the uninjured hemisphere. Our studies strongly suggest that the same activity-dependent mechanisms that ensure optimal CST development under normal conditions can become maladaptive after perinatal injury to the CS system.

With an initial bilateral organization, the typical-developing CST eliminates most ipsilateral spinal projections (Figure 1). As discussed above, the initial density of ipsilateral CST projections is regulated, in part, by a genetic mechanism (EphA4) and the subsequent reduction in ipsilateral projections reflects an activitydependent refinement process (12). Figure 5A shows the normal contralateral (top) and ipsilateral (bottom) projections. When the activity of the CS system in one hemisphere is reduced, by intracortical infusion of muscimol, the affected hemisphere is not able to establish its normal projection pattern contralaterally (Figure 5B, top). In a reciprocal manner, the ipsilateral projections of the CS system in the other hemisphere are better able to compete with the less active CST for synaptic connections with spinal cord neurons (Figure 5B, bottom). In these experiments, CST axons from 


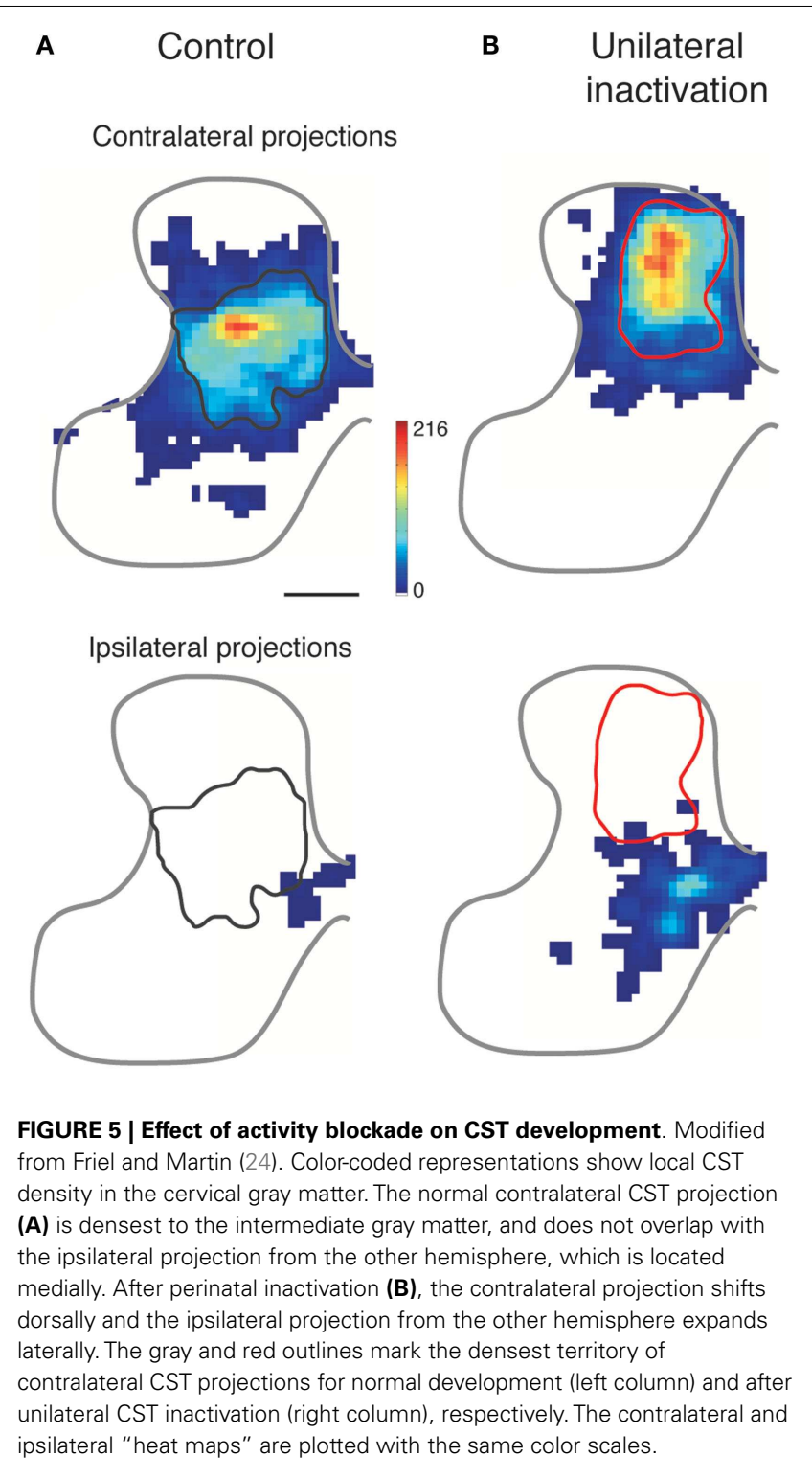

only a small portion of the forelimb area of motor cortex were labeled. Nevertheless, comparison of the control and inactivated animal revealed a 3.6-fold increase in the spatial extent of the ipsilateral projections with a peak approximately $1 / 3$ that of the contralateral projection. Thus, the active CS system maintains, and likely strengthens, its early bilateral organization (Figure 5B, bottom). The incursion of abundant CST outgrowth into the ipsilateral spinal gray matter greatly impedes development of the less active CST. The contralateral axons of the less active CST are prevented from typical outgrowth and synapse formation $(24,40)$. Further, there is a dorsal shift for the inactivated CST (24, 40, 41). We do not yet know the mechanism of this circuit change. However, it is apt to be functionally significant because this dorsal area of the dorsal horn is more concerned with the processing of somatic sensory information than motor output (28). Because of this location, the impaired CST may have reduced access to spinal

\section{A Aberrant ipsilateral CST}
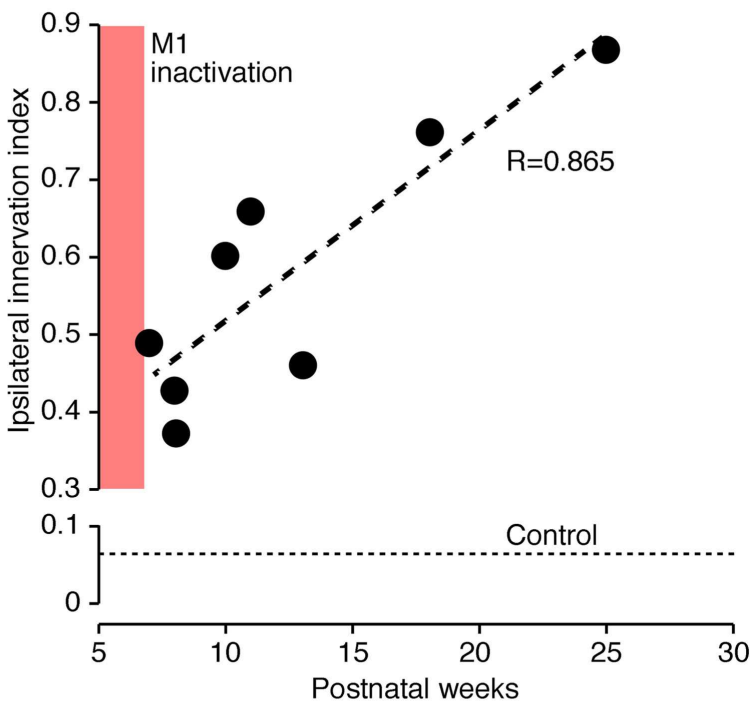

B Vicious circle model

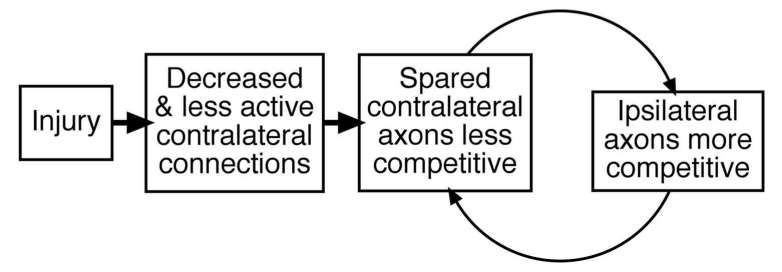

FIGURE 6 | Vicious circle hypothesis (42). (A) Aberrant ipsilateral corticospinal terminations further develop postnatally. The graph plots the ratio of local density of ipsilateral and contralateral CST terminations. (B) Hypothesis of progressive decline of less active connections and complementary augmentation of more active connections.

motor circuits and, as a consequence, is much less functional. The question remains in the animal model if this aberrant dorsal CST projection is maladaptive. By not targeting their proper spinal circuits, this projection could underlie aberrant contralateral control, such as spasticity, incoordination, and reflex radiation.

As development progresses, the ipsilateral CST from the active side continues to establish connections at the expense of the contralateral projection from the inactive side. We showed that this process progresses even after activity is restored to the previously silenced side (Figure 6). We propose that perinatal injury before elimination of early ipsilateral CST projections creates a "vicious circle" of further loss of injured and gain of spared CST fibers (42). This is due to impaired capacity of the injured side to maintain connections, and a concomitant robust reactive increase in ipsilateral CST projections from the undamaged hemisphere. The underlying mechanism may be activity-dependent synaptic competition, with a loss of competitive ability of the injured side and a gain of competitive ability by the undamaged (or less damaged) side.

We equate the less active CST in our model with the damaged CST after a lesion. The affected limb is used less than normal, 
thus the surviving fibers of the CST serving that limb would be expected to be less active. There also would be reduced afferent feedback because of the paucity of movements, and possibly reorganization of proprioceptive inputs to the spinal cord $(43,44)$. Conversely, the unaffected (or less affected) limb is used more and the undamaged CST would be expected to be more active. Thus, experimentally manipulated activity changes both inform mechanism (e.g., activity may be more important than the physical loss of connections due to damage) and are also a reasonable model for what happens after a unilateral lesion. Damage to one hemisphere, and the associated lack of limb use, can thus lead to a dual vulnerability. The damaged side loses much of its contralateral connections to injury, while the undamaged (or less damaged) side "fills in" these denervated territories with ipsilateral misprojections and further constrains development of the remaining contralateral CST projections.

We hypothesize that the aberrant, and possibly maladaptive, ipsilateral CST in people with hemiplegic CP reflects a basic mechanism for synaptic stabilization that goes awry after injury. With injury, more robust than loss of activity as in our animal model, the loss of connections and lack of affected limb use makes this side less able to compete for synaptic connections with spinal motor circuits than the uninjured system. By contrast, the lessaffected CST concomitantly gains synaptic competitive ability. When damage occurs after the early ipsilateral CST projections are eliminated, reduced synaptic competition may continue to play an important role in the evolving motor impairment. In maturity, loss of connections of the damaged system is counterbalanced by a small reactive increase in spared ipsilateral CST projections from the undamaged hemisphere $(45,46)$ and a reactive increase in proprioceptive afferent projections $(47,48)$. At this point after an injury in maturity, denervated spinal circuits would be driven more by afferent fibers and ipsilateral CST projections. This too likely results in motor impairment.

\section{INTERVENTIONAL STRATEGIES}

Knowledge of the pathophysiology of the developing CST after injury informs interventional strategies for protecting the damaged system from further loss of connections and function during development. This knowledge is also helpful in devising strategies for repairing aberrant CS circuits once development has taken place. As discussed, a well-known misprojection of the CST in patients with hemiplegic CP is development of ipsilateral misprojections. Eyre et al. (49) have shown that perinatal injury results in the progressive loss of the damaged CST and maintenance and possible strengthening of the ipsilateral CST; this is akin to the vicious circle discussed above (42). The net outcome is that the spared side develops an aberrant bilateral CST. We infer from our genetic model [Figures 2 and 3; (17)] that one consequence of these bilateral misprojections in CP is mirror movements (2). Other misprojections are likely to play a role in impaired intralimb coordination, reflex radiations, and spasticity. We hypothesize that during the period when the ipsilateral projections are strengthening after perinatal injury, activity-dependent competition is "out of control" driving down contralateral function of the affected side and establishing stronger ipsilateral projections from the non-affected side.
Using the cat model described in the previous section, we aimed to repair the aberrant pattern of connections and restore function by harnessing activity-dependent processes. According to our competition hypothesis, either improving the capacity of the injured system to compete for spinal synaptic connections or diminishing the unaffected system to compete, should help restore a more normal CST pattern of connections and improve function. We tackled both approaches and achieved differential success that can be explained on the basis of a dosing effect.

In two separate studies, we showed that promoting the activity of the injured system (26) or decreasing the activity of the undamaged system (24) immediately after the period of injury leads to partial repair of the CST and restoration of skilled motor function. These remarkably effective activity-based interventions provide proof-of-principle of the capacity to harness activity after developmental impairment to repair CST connections and function. We next took a more clinically minded approach and used constraint of the less-impaired limb at two time points, with and without behavioral training, to determine if behavioral approaches are similarly effective. We examined three conditions: (1) constraint immediately after the impairment; (2) constraint immediately after the impairment plus intensive motor training (reach to grasp); and (3) delayed constraint with intensive motor training. It is important to recognize that the effects of early developmental impairment [e.g., Figure 6B; CST abnormalities and motor control impairments; $(24,50)]$ are permanent without further intervention. Remarkably, whereas all three interventions result in comparable repair of the contralateral CST (Figure 7A), there were differences in the motor cortex motor maps and spinal interneuronal function that accounted for whether or not animals showed behavioral recovery.

With constraint and intensive training both initiated early in development (Figure 7), there was restoration of the motor cortex motor map; a complete representation of contralateral forelimb joints was present (Figure 7B) (41). Thus, representational plasticity, which occurs throughout the animals lifetime $(35,51)$, helps to provide an effective substrate for contralateral limb control. Although the absolute numbers of spinal cholinergic interneurons were not different, side-to-side, we also observed relative increases in spinal cholinergic interneurons (Figure 7C; boxed region compares interneurons in the intermediate zone). These interneurons may play a key role in relaying CST signals to motoneurons (5255). Together, the motor map and interneuronal changes support behavioral recovery. Without intensive training (Figure 7, middle column), the motor cortex motor map failed to show representational plasticity; the motor map was essentially lost (41). Further, interneuronal numbers did not improve. Together, the absence of the map and insufficient interneuronal changes could have prevented behavioral recovery. Clearly, restraint alone is not therapeutic; it needs to be combined with training.

Importantly, delayed intervention into the feline equivalent of young adolescence led to restoring the motor map but not cholinergic spinal interneurons [Figure 7, third column; (41)]. Since this animal group did not recover function, but did show repaired CST projections and M1 motor map, it shows the importance of spinal circuitry and possibly other pathways (e.g., rubrospinal tract) in normal and recovered function. In the cat, spinal cholinergic 


\section{Early training Restraint alone Delayed training}

\section{A Axons}
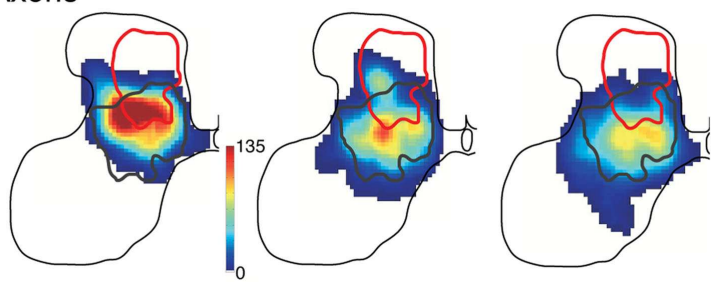

B Motor map
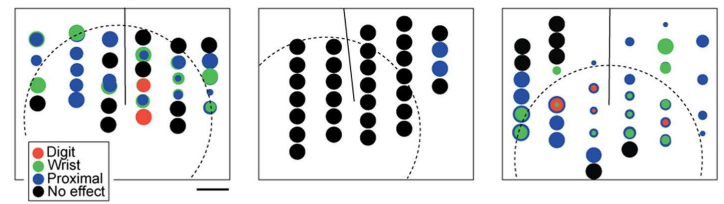

c ChAT interneurons
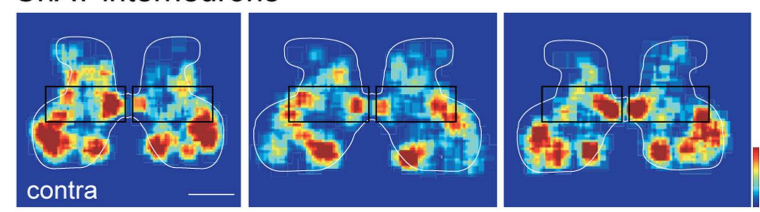

D Forward distance (cm)

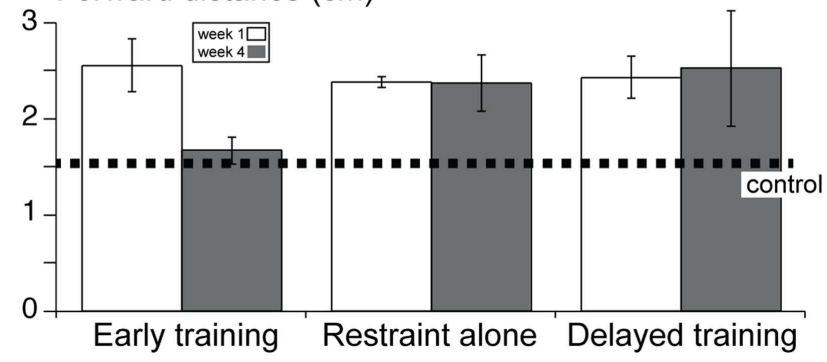

FIGURE 7 | Effect of constraint of the unimpaired limb on CST projections, the motor cortex motor map, spinal cholinergic interneurons, and skilled behavior. Modified from Friel et al. (41). (A) Distribution of CST projections in the cervical enlargement. Density of projections is represented by a color scale, with red corresponding to the densest projections. All "heat maps" are plotted with the same color scale. The red line corresponds to the aberrant dorsal projection of the inactivated CST (as shown in Figure 6), whereas the dark gray line is the normal distribution (Figure 6). (B) Motor cortex motor map. Each site examined for a motor response with stimulation is marked by a circle. The threshold for evoking a contralateral motor response is represented inversely by circle diameter. Black corresponds to no response sites. (C) Color-coded representations of local cholinergic interneuron density. Boxes highlight differences in relative density. Contralateral compared with ipsilateral density is greatest for early training. (D) Forward distance is a measure of reaching accuracy. The dotted line marks normal performance. Over-reaching is characteristic at 1 week for all groups. Only the early trained group shows recovery to normal distances; performance of the other groups remains errant.

interneurons develop during the same period when the CST connections are being refined (53). We hypothesize that there is a critical period for spinal cholinergic interneuron development, under CST activity control (41). The capacity to restore this interneuronal function is restricted to the early intervention period. This demonstrates the importance of repair strategies that take other neural systems into account. The rubrospinal system is another potentially important system that should be considered in repair after perinatal damage.

Several studies have demonstrated efficacy of activity-based therapies for children with hemiplegic CP (56-61). These therapies - either constraining the less-affected upper limb and intensively training the affected upper limb, or intensively training bimanual upper limb movements - improve hand function in children with hemiplegia. Since intensive therapy requires children to engage and follow instructions, these therapies are typically delivered to school-aged children. It is hypothesized that, just as in our cat model, these therapies increase activity of the impaired side, thus increasing the competitive strength of the impaired side against the less-impaired side. Although these activity-based therapies improve upper limb control, function is not fully restored. Hand function is still most often substantially impaired compared with typically developing children. Our studies in the cat model indicate that early intervention, before the less-impaired CST secures a strong competitive advantage over the impaired CST, may be necessary for mitigating the motor dysfunction of CP.

Although clinical application of activity-based therapies is challenging in young children, there have been efforts to translate therapy to this population. Gordon et al. (62) have developed a caregiver-driven bimanual therapy for toddlers that significantly improved bimanual hand use and performance of functional tasks. Several studies have indicated that enriched environments that encourage infants to move and reach can improve motor outcomes in very young children who have, or are at risk of developing, CP (63-65).

Non-invasive brain stimulation may be a viable strategy for balancing activity-dependent competition between the two sides of the brain after unilateral brain injury. Repetitive transcranial magnetic stimulation (rTMS) has been shown to boost the amount of motor improvements seen after gait or hand training (66, 67). Recently, transcranial direct current stimulation (tDCS) has emerged as a preferred method of non-invasive brain stimulation due to its excellent safety profile, portability, and low cost. tDCS has been tested in a small number of children with hemiplegia, and has been shown to be safe and tolerable (66). Non-invasive brain stimulation may emerge as a potential therapy for very young children, to balance interhemispheric competition before the damaged side loses its foothold in the developing motor system. However, safety is an important concern that must be evaluated further. The use of brain modeling is likely to be an important step in determining the safety of brain stimulation in young children $(66,68)$.

\section{PERSPECTIVES AND CONCLUSION}

We envisage two distinct periods of intervention, where repair of the damaged CS system can occur. Very early during development, during the so-called critical period, activity-based approaches can have a neuroprotective effect by enhancing the ability of the damaged CST to establish spinal connections. This is likely the most effective period since it is directed to facilitate spinal circuit development. Extrapolating from the cat data, in the human this 
would correspond to the first 6-12 postnatal months (19). Later in development, after aberrant CST connections are established and become permanent if not treated further, there is still a robust capacity to repair. CST growth to original targets and partial elimination of aberrant ipsilateral misprojections can occur. Indeed, intact CST axons are capable of sprouting into the denervated side of the spinal cord in maturity $(45,46,69)$. However, the efficacy is strongly reduced after the critical period and is insufficient to restore significant function unless promoted. There also is robust representational plasticity of the motor cortex (41). Importantly, in our model, behavioral therapies do not appear to be effective in repairing aberrant spinal interneuronal circuits later in development. It is plausible that direct activity manipulations, such as motor cortex stimulation $(45,46)$, are needed to coax spinal interneurons to improve function.

A critical question is if the ipsilateral CST after unilateral development lesion can be adaptive? By themselves, ipsilateral CST projections target different sets of spinal cord laminae compared with their contralateral counterparts $(45,69)$. As a consequence, different somatic sensory and motor circuits will be engaged by the ipsilateral and contralateral tracts. This points to limitations in function but not necessarily maladaptive functions. Further, the ipsilateral projection in concert with the contralateral projection leads to bilateral control, and impairments such as mirror movements. However, it has been shown in maturity that strengthening spared ipsilateral CST axons actually leads to functional improvements in a rat model of unilateral CST lesion $(46,70)$. We think that these findings point to a different culprit. It is not the ipsilateral CST that is maladaptive but rather the consequential loss of the contralateral projection due to reduced ability to compete for synaptic connections in the spinal cord. Furthermore, if the loss of the contralateral projection also triggers afferent fiber sprouting during development, as it does in maturity (48), then there are additional potential sources of maladaptive spinal inputs. It may be necessary to repair multiple neural systems CST, spinal interneurons, afferent fibers, and rubrospinal fibers, to name four - before motor function can be fully restored. Our interventional approaches that harness neural activity-dependent processes are highly effective in an animal model of hemiplegic CP. These approaches are minimally invasive and are poised for translation to the human.

\section{ACKNOWLEDGMENTS}

This work was supported by the NIH (JHM: R01NS079569 2R01NS064004).

\section{REFERENCES}

1. Sanger TD, Chen D, Delgado MR, Gaebler-Spira D, Hallett M, Mink JW, et al. Definition and classification of negative motor signs in childhood. Pediatrics (2006) 118:2159-67. doi:10.1542/peds.2005-3016

2. Kuhtz-Buschbeck JP, Sundholm LK, Eliasson AC, Forssberg H. Quantitative assessment of mirror movements in children and adolescents with hemiplegic cerebral palsy. Dev Med Child Neurol (2000) 42:728-36. doi:10.1111/j.14698749.2000.tb00034.x

3. Ellis MD, Acosta AM, Yao J, Dewald JP. Position-dependent torque coupling and associated muscle activation in the hemiparetic upper extremity. Exp Brain Res (2007) 176:594-602. doi:10.1007/s00221-006-0637-x

4. Cahill-Rowley K, Rose J. Etiology of impaired selective motor control: emerging evidence and its implications for research and treatment in cerebral palsy. Dev Med Child Neurol (2014) 56:522-8. doi:10.1111/dmcn.12355
5. Sukal-Moulton T, Krosschell KJ, Gaebler-Spira DJ, Dewald JP. Motor impairment factors related to brain injury timing in early hemiparesis. Part I: expression of upper-extremity weakness. Neurorehabil Neural Repair (2014) 28:13-23. doi: $10.1177 / 1545968313500564$

6. Sukal-Moulton T, Krosschell KJ, Gaebler-Spira DJ, Dewald JP. Motor impairments related to brain injury timing in early hemiparesis. Part II: abnormal upper extremity joint torque synergies. Neurorehabil Neural Repair (2014) 28:24-35. doi:10.1177/1545968313497829

7. Martin JH, Ghez C. Red nucleus and motor cortex: parallel motor systems for the initiation and control of skilled movement. Behav Brain Res (1988) 28:217-23. doi:10.1016/0166-4328(88)90099-X

8. Cheney PD, Fetz EE, Mewes K. Neural mechanisms underlying corticospinal and rubrospinal control of limb movements. Prog Brain Res (1991) 87:213-52. doi:10.1016/S0079-6123(08)63054-X

9. Arlotta P, Molyneaux BJ, Chen J, Inoue J, Kominami R, Macklis JD. Neuronal subtype-specific genes that control corticospinal motor neuron development in vivo. Neuron (2005) 45:207-21. doi:10.1016/j.neuron.2004.12.036

10. Molyneaux BJ, Arlotta P, Hirata T, Hibi M, Macklis JD. Fezl is required for the birth and specification of corticospinal motor neurons. Neuron (2005) 47:817-31. doi:10.1016/j.neuron.2005.08.030

11. Woodworth MB, Custo Greig L, Kriegstein AR, Macklis JD. SnapShot: cortical development. Cell (2012) 151(918-918):e911. doi:10.1016/j.cell.2012. 10.004

12. Martin J, Friel K, Salimi I, Chakrabarty S. Corticospinal Development. In: Squire L, editor. Encyclopedia of Neuroscience. Oxford: Academic Press (2009). p. 203-14.

13. Mai J, Fok L, Gao H, Zhang X, Poo MM. Axon initiation and growth cone turning on bound protein gradients. J Neurosci (2009) 29:7450-8. doi:10.1523/ JNEUROSCI.1121-09.2009

14. Dottori M, Hartley L, Galea M, Paxinos G, Polizzotto M, Kilpatrick T, et al. EphA4 (Sek1) receptor tyrosine kinase is required for the development of the corticospinal tract. Proc Natl Acad Sci U S A (1998) 95:13248-53. doi:10.1073/ pnas.95.22.13248

15. Kullander K, Croll SD, Zimmer M, Pan L, McClain J, Hughes V, et al. Ephrin$\mathrm{B} 3$ is the midline barrier that prevents corticospinal tract axons from recrossing, allowing for unilateral motor control. Genes Dev (2001) 15:877-88. doi:10.1101/gad.868901

16. Paixao S, Balijepalli A, Serradj N, Niu J, Luo W, Martin JH, et al. EphrinB3/EphA4-mediated guidance of ascending and descending spinal tracts. Neuron (2013) 80:1407-20. doi:10.1016/j.neuron.2013.10.006

17. Serradj N, Paixao S, Sobocki T, Feinberg M, Klein R, Kullander K, et al. EphA4mediated ipsilateral corticospinal tract misprojections are necessary for bilateral voluntary movements but not bilateral stereotypic locomotion. J Neurosci (2014) 34:5211-21. doi:10.1523/JNEUROSCI.4848-13.2014

18. Li Q, Martin JH. Postnatal development of differential projections from the caudal and rostral motor cortex subregions. Exp Brain Res (2000) 134:187-98. doi:10.1007/s002210000454

19. Eyre JA, Miller S, Clowry GJ, Conway EA, Watts C. Functional corticospinal projections are established prenatally in the human foetus permitting involvement in the development of spinal motor centres. Brain (2000) 123:51-64. doi:10.1093/brain/123.1.51

20. Eyre JA, Taylor JP, Villagra F, Smith M, Miller S. Evidence of activity-dependent withdrawal of corticospinal projections during human development. Neurology (2001) 57:1543-54. doi:10.1212/WNL.57.9.1543

21. Brosamle C, Schwab ME. Cells of origin, course, and termination patterns of the ventral, uncrossed component of the mature rat corticospinal tract. J Comp Neurol (1997) 386:293-303. doi:10.1002/(SICI)1096-9861(19970922) 386:2<293::AID-CNE9>3.0.CO;2-X

22. Rosenzweig ES, Brock JH, Culbertson MD, Lu P, Moseanko R, Edgerton VR, et al. Extensive spinal decussation and bilateral termination of cervical corticospinal projections in rhesus monkeys. J Comp Neurol (2009) 513:151-63. doi:10.1002/cne.21940

23. Asante CO, Chu A, Fisher M, Benson L, Beg A, Scheiffele P, et al. Cortical control of adaptive locomotion in wild-type mice and mutant mice lacking the ephrinEph effector protein \{alpha\}2-chimaerin. J Neurophysiol (2010) 104:3189-202. doi:10.1152/jn.00671.2010

24. Friel K, Martin JH. Bilateral activity-dependent interactions in the developing corticospinal system. J Neurosci (2007) 27:11083-90. doi:10.1523/JNEUROSCI. 2814-07.2007 
25. Martin JH, Choy M, Pullman S, Meng Z. Corticospinal development depends on experience. J Neurosci (2004) 24:2122-32. doi:10.1523/JNEUROSCI.461603.2004

26. Salimi I, Friel KM, Martin JH. Pyramidal tract stimulation restores normal corticospinal tract connections and visuomotor skill after early postnatal motor cortex activity blockade. J Neurosci (2008) 28:7426-34. doi:10.1523/JNEUROSCI. 1078-08.2008

27. Martin JH, Lee S. Activity-dependent competition between developing corticospinal terminations. Neuroreport (1999) 10:2277-82. doi:10.1097/00001756199908020-00010

28. Kuypers HGJM. Anatomy of the descending pathways. In: Brookhart JM, Mountcastle VB, editors. Handbook of Physiology, Neurophysiology. (Vol. II), Bethesda, MD: American Physiological Society (1981). p. 597-666.

29. Yeo SS, Jang SH. Changes in red nucleus after pyramidal tract injury in patients with cerebral infarct. NeuroRehabilitation (2010) 27:373-7. doi:10.3233/NRE2010-0622

30. Ruber T, Schlaug G, Lindenberg R. Compensatory role of the cortico-rubrospinal tract in motor recovery after stroke. Neurology (2012) 79:515-22. doi:10.1212/WNL.0b013e31826356e8

31. Ulfig N, Chan WY. Differential expression of calcium-binding proteins in the red nucleus of the developing and adult human brain. Anat Embryol (Berl) (2001) 203:95-108. doi:10.1007/s004290000147

32. Williams PT, Kim S, Martin JH. Postnatal maturation of the red nucleus motor map depends on rubrospinal connections with forelimb motor pools. J Neurosci (2014) 34:4432-41. doi:10.1523/JNEUROSCI.5332-13.2014

33. Monfils MH, Plautz EJ, Kleim JA. In search of the motor engram: motor map plasticity as a mechanism for encoding motor experience. Neuroscientist (2005) 11:471-83. doi:10.1177/1073858405278015

34. Bruce IC, Tatton WG. Synchronous development of motor cortical output to different muscles in the kitten. Exp Brain Res (1980) 40:349-53.

35. Chakrabarty S, Martin JH. Postnatal development of the motor representation in primary motor cortex. J Neurophysiol (2000) 84:2582-94.

36. Barrett P, Bateson P. The development of play in cats. Behaviour (1978) 66:106-20. doi:10.1163/156853978X00422

37. Martin P, Bateson P. The ontogeny of locomotor play behavior in the domestic cat. Anim Behav (1985) 33:502-10. doi:10.1016/S0003-3472(85)80073-7

38. Williams PT, Martin JH. Development of the Red Nucleus Motor Map Depends on Motor Cortex Activity During a Key Postnatal Period. Program No. 185.20. Neuroscience 2012 Abstracts. New Orleans, LA: Society for Neuroscience (2012).

39. Williams PT, Kim S, Zuelke D, Chang A, Martin JH. Motor Cortex Inactivity During a Key Period of Development Permanently Alters the Organization of the Rubrospinal System. Program No. 72.13. Neuroscience 2014 Abstracts. Washington, DC: Society for Neuroscience (2014).

40. Friel K, Martin JH. Role of sensory-motor cortex activity in postnatal development of corticospinal axon terminals in the cat. JComp Neurol (2005) 485:43-56. doi: $10.1002 / \mathrm{cne} .20483$

41. Friel K, Chakrabarty S, Kuo HC, Martin J. Using motor behavior during an early critical period to restore skilled limb movement after damage to the corticospinal system during development. J Neurosci (2012) 32:9265-76. doi:10.1523/JNEUROSCI.1198-12.2012

42. Martin JH. Systems neurobiology of restorative neurology and future directions for repair of the damaged motor systems. Clin Neurol Neurosurg (2012) 114:515-23. doi:10.1016/j.clineuro.2012.01.011

43. Gibson CL, Arnott GA, Clowry GJ. Plasticity in the rat spinal cord seen in response to lesions to the motor cortex during development but not to lesions in maturity. Exp Neurol (2000) 166:422-34. doi:10.1006/exnr.2000.7511

44. Clowry GJ, Davies BM, Upile NS, Gibson CL, Bradley PM. Spinal cord plasticity in response to unilateral inhibition of the rat motor cortex during development: changes to gene expression, muscle afferents and the ipsilateral corticospinal projection. Eur J Neurosci (2004) 20:2555-66. doi:10.1111/j.1460-9568.2004. 03713.x

45. Brus-Ramer M, Carmel JB, Chakrabarty S, Martin JH. Electrical stimulation of spared corticospinal axons augments connections with ipsilateral spinal motor circuits after injury. J Neurosci (2007) 27:13793-801. doi:10.1523/JNEUROSCI. 3489-07.2007

46. Carmel JB, Berol L, Brus-Ramer M, Martin JH. Chronic electrical stimulation of the intact corticospinal system after unilateral injury restores skilled locomotor control and promotes spinal axon outgrowth. J Neurosci (2010) 30:10918-26. doi:10.1523/JNEUROSCI.1435-10.2010

47. Jiang Y, Martin JH. Competition and Compensation between the Corticospinal and Spinal Mechanoafferent Systems. Program No. 582.12. Neuroscience 2012 Abstracts. New Orleans, LA: Society for Neuroscience (2012).

48. Tan AM, Chakrabarty S, Kimura H, Martin JH. Selective corticospinal tract injury in the rat induces primary afferent fiber sprouting in the spinal cord and hyperreflexia. J Neurosci (2012) 32:12896-908. doi:10.1523/JNEUROSCI.645111.2012

49. Eyre JA, Smith M, Dabydeen L, Clowry GJ, Patacchi E, Battini R, et al. Is hemiplegic cerebral palsy equivalent to amblyopia of the corticospinal system? Ann Neurol (2007) 62:493-503. doi:10.1002/ana.21108

50. Friel KM, Drew T, Martin JH. Differential activity-dependent development of corticospinal control of movement and final limb position during visuallyguided locomotion. J Neurophysiol (2007) 97:3396-406. doi:10.1152/jn.00750. 2006

51. Chakrabarty S, Martin JH. Motor but not sensory representation in motor cortex depends on postsynaptic activity during development and in maturity. J Neurophysiol (2005) 94:3192-8. doi:10.1152/jn.00424.2005

52. Miles GB, Hartley R, Todd AJ, Brownstone RM. Spinal cholinergic interneurons regulate the excitability of motoneurons during locomotion. Proc Natl Acad Sci U S A (2007) 104:2448-53. doi:10.1073/pnas.0611134104

53. Chakrabarty S, Shulman B, Martin JH. Activity-dependent codevelopment of the corticospinal system and target interneurons in the cervical spinal cord. J Neurosci (2009) 29:8816-27. doi:10.1523/JNEUROSCI.0735-09.2009

54. Zagoraiou L, Akay T, Martin JF, Brownstone RM, Jessell TM, Miles GB. A cluster of cholinergic premotor interneurons modulates mouse locomotor activity. Neuron (2009) 64:645-62. doi:10.1016/j.neuron.2009.10.017

55. Chakrabarty S, Martin JH. Postnatal development of a segmental switch enables corticospinal tract transmission to spinal forelimb motor circuits. J Neurosci (2010) 30:2277-88. doi:10.1523/JNEUROSCI.5286-09.2010

56. de Brito Brandao M, Gordon AM, Mancini MC. Functional impact of constraint therapy and bimanual training in children with cerebral palsy: a randomized controlled trial. Am J Occup Ther (2012) 66:672-81. doi:10.5014/ajot 2012.004622

57. Brandao MB, Ferre C, Kuo HC, Rameckers EA, Bleyenheuft Y, Hung YC, et al. Comparison of structured skill and unstructured practice during intensive bimanual training in children with unilateral spastic cerebral palsy. Neurorehabil Neural Repair (2013) 28:452-61. doi:10.1177/1545968313516871

58. Deppe W, Thuemmler K, Fleischer J, Berger C, Meyer S, Wiedemann B. Modified constraint-induced movement therapy versus intensive bimanual training for children with hemiplegia - a randomized controlled trial. Clin Rehabil (2013) 27:909-20. doi:10.1177/0269215513483764

59. Dong VA, Tung IH, Siu HW, Fong KN. Studies comparing the efficacy of constraint-induced movement therapy and bimanual training in children with unilateral cerebral palsy: a systematic review. Dev Neurorehabil (2013) 16:133-43. doi:10.3109/17518423.2012.702136

60. Sakzewski L, Gordon A, Eliasson AC. The state of the evidence for intensive upper limb therapy approaches for children with unilateral cerebral palsy. JChild Neurol (2014) 29:1077-90. doi:10.1177/0883073814533150

61. Sakzewski L, Ziviani J, Boyd RN. Efficacy of upper limb therapies for unilateral cerebral palsy: a meta-analysis. Pediatrics (2014) 133:e175-204. doi:10.1542/ peds.2013-0675

62. Ferre CL, Brandao MB, Hung YC, Carmel JB, Gordon AM. Feasibility of caregiver-directed home-based hand-arm bimanual intensive training: a brief report. Dev Neurorehabil (2014):1-6. doi:10.3109/17518423.2014.948641

63. Al-Oraibi S, Eliasson AC. Implementation of constraint-induced movement therapy for young children with unilateral cerebral palsy in Jordan: a home-based model. Disabil Rehabil (2011) 33:2006-12. doi:10.3109/09638288.2011.555594

64. Prosser LA, Ohlrich LB, Curatalo LA, Alter KE, Damiano DL. Feasibility and preliminary effectiveness of a novel mobility training intervention in infants and toddlers with cerebral palsy. Dev Neurorehabil (2012) 15:259-66. doi: $10.3109 / 17518423.2012 .687782$

65. Morgan C, Novak I, Badawi N. Enriched environments and motor outcomes in cerebral palsy: systematic review and meta-analysis. Pediatrics (2013) 132:e735-46. doi:10.1542/peds.2012-3985

66. Kirton A, Chen R, Friefeld S, Gunraj C, Pontigon AM, Deveber G. Contralesional repetitive transcranial magnetic stimulation for chronic hemiparesis in 
subcortical paediatric stroke: a randomised trial. Lancet Neurol (2008) 7:507-13. doi:10.1016/S1474-4422(08)70096-6

67. Gillick BT, Krach LE, Feyma T, Rich TL, Moberg K, Thomas W, et al. Primed lowfrequency repetitive transcranial magnetic stimulation and constraint-induced movement therapy in pediatric hemiparesis: a randomized controlled trial. Dev Med Child Neurol (2014) 56:44-52. doi:10.1111/dmcn.12243

68. Kessler SK, Minhas P, Woods AJ, Rosen A, Gorman C, Bikson M. Dosage considerations for transcranial direct current stimulation in children: a computational modeling study. PLoS One (2013) 8:e76112. doi:10.1371/journal.pone.0076112

69. Carmel JB, Kimura H, Berrol LJ, Martin JH. Motor cortex electrical stimulation promotes axon outgrowth to brain stem and spinal targets that control the forelimb impaired by unilateral corticospinal injury. Eur J Neurosci (2013) 37(7):1090-102. doi:10.1111/ejn.12119

70. Carmel JB, Kimura H, Martin JH. Electrical stimulation of motor cortex in the uninjured hemisphere after chronic unilateral injury promotes recovery of skilled locomotion through ipsilateral control. J Neurosci (2014) 34:462-6. doi:10.1523/JNEUROSCI.3315-13.2014
Conflict of Interest Statement: The authors declare that the research was conducted in the absence of any commercial or financial relationships that could be construed as a potential conflict of interest.

Received: 10 August 2014; accepted: 22 October 2014; published online: 24 November 2014.

Citation: Friel KM, Williams PTJA, Serradj N, Chakrabarty S and Martin JH (2014) Activity-based therapies for repair of the corticospinal system injured during development. Front. Neurol. 5:229. doi: 10.3389/fneur.2014.00229

This article was submitted to Neuropediatrics, a section of the journal Frontiers in Neurology.

Copyright $\odot 2014$ Friel, Williams, Serradj, Chakrabarty and Martin. This is an openaccess article distributed under the terms of the Creative Commons Attribution License (CC BY). The use, distribution or reproduction in other forums is permitted, provided the original author(s) or licensor are credited and that the original publication in this journal is cited, in accordance with accepted academic practice. No use, distribution or reproduction is permitted which does not comply with these terms. 